\title{
Review of: "Mutated neuronal voltage-gated CaV2.1 channels causing familial hemiplegic migraine 1 increase the susceptibility for cortical spreading depolarization and seizures and worsen outcome after experimental traumatic brain injury"
}

Andrea Nistri

Potential competing interests: The author(s) declared that no potential competing interests exist.

The MS by Terpolilli et al reports data concerning cortical spreading depression, seizures and behavioural changes in a mouse trangeneic model of familial hemiplegic migraine. Such a mouse model has been largely investigated by many labs before and facilitated CDS and related symptoms have been described earlier. Indeed,. former studies in this area are extensively quoted by the authors. From this point of view the current MS is not particularly novel even if the experimental results have been clearly detailed and illustrated. In practice, the present study adds some information to our current knowledge of CNS pathology of this mouse model. However, the only new data are those indicating that skull surgery trauma elicited stronger and longer lasting excitation of the model mouse brain.

Where the MS needs refocussing is the issue of "mechanisms" for this phenomenon. Unfortunately there are no data to show wheter there was enhanced excitatory synaptic transmission or deficit of synaptic inhibition followign the trauma, or whether certain receptors or channels had been impacted by the trauma for a short or long time. If data pertaining these issues are not available to strengthen the MS, it is suggested to avoid referrring to mechanisms that remain unexplored and, thus, unknown. 